\title{
Influence of Variant of Concern B.1.1.7 on In-Hospital Mortality in SARS-CoV-2 Hospitalized Patients
}

\author{
Nele Gessler ${ }^{1,2,3, \# *}$, Melanie A Gunawardene ${ }^{1 \#}$, Peter Wohlmuth ${ }^{2}$, Dirk Arnold ${ }^{4}$, Martin \\ Bachmann $^{5}$, Juergen Behr ${ }^{6}$, Berthold Bein ${ }^{7}$, Martin Bergmann ${ }^{8}$, Christian Gloeckner ${ }^{9}$, Klaus \\ Herrlinger $^{10}$, Thomas Hoelting ${ }^{8}$, Ulrich Frank Pape ${ }^{11}$, Lorenz Nowak ${ }^{12}$, Tino Schnitgerhans ${ }^{10}$, \\ Ruediger Schreiber ${ }^{13}$, Axel Stang ${ }^{3,14}$, Claas Wesseler ${ }^{5}$, Stephan Willems ${ }^{1}$, Sebastian Wirtz ${ }^{15}$, \\ Hanna Nugent ${ }^{3}$ and Christoph U Herborn ${ }^{2,3,16}$
}

${ }^{1}$ Department of Cardiology and Internal intensive care medicine, Asklepios Hospital St. Georg, Hamburg, Germany

${ }^{2}$ Asklepios Proresearch, Research institute, Hamburg, Germany

${ }^{3}$ Semmelweis University, Budapest, Hungary

${ }^{4}$ Department of Hematology, Oncology, Palliative Care Medicine and Rheumatology, Asklepios Hospital Altona, Hamburg, Germany

${ }^{5}$ Department of Intensive and Ventilatory Medicine, Asklepios Hospital Harburg, Hamburg, Germany

${ }^{6}$ Department of Pneumology, Member of the German Center for Lung Research, Asklepios Hospital Munich-Gauting, Gauting, Germany ${ }^{7}$ Department of Anesthesiology, Intensive care, Emergency medicine and Pain management, Asklepios Hospital St. Georg, Hamburg, Germany

${ }^{8}$ Department of Internal Medicine - Cardiology and Pneumology, Asklepios Hospital Wandsbek, Hamburg, Germany

${ }^{9}$ Department of Internal Medicine, Asklepios Hospital Oberviechtach, Oberviechtach, Germany

${ }^{10}$ Department of Internal medicine-Gastroenterology, Asklepios Hospital Nord-Heidberg, Hamburg, Germany

${ }^{11}$ Department of Internal medicine - Gastroenterology, Asklepios Hospital St. Georg, Hamburg, Germany

${ }^{12}$ Department of Pneumology and Intensive Care Medicine, Member of the German Center for Lung Research, Asklepios Hospital MunichGauting, Gauting, Germany

${ }^{13}$ Department Anesthesiology and Intensive care medicine, Asklepios Hospital West, Hamburg, Germany

${ }^{14}$ Department of Oncology and Palliative Care Medicine, Asklepios Hospital Barmbek, Hamburg, Germany

${ }^{15}$ Department of Anesthesiology and Intensive care Medicine, Asklepios Hospital Barmbek, Hamburg, Germany

${ }^{16}$ Asklepios hospitals GmbH \& Co. KGaA, Hamburg, Germany

\#These authors contributed equally to this work

*Corresponding author: Nele Gessler, Department of Cardiology and Internal intensive care medicine, Asklepios Hospital St. Georg, Hamburg, Germany

ARTICLE INFO

Received: 絈 August 16, 2021

Published: 幽 August 24, 2021

\section{ABSTRACT}

Citation: Nele Gessler, Melanie A Gunawardene, Peter Wohlmuth, Dirk Arnold, Martin Bachmann, et al., Influence of Variant of Concern B.1.1.7 on In-Hospital Mortality in SARSCov-2 Hospitalized Patients. Biomed J Sci \& Tech Res 38(2)-2021. BJSTR. MS.ID.006138. 


\section{Introduction}

Since December 2020, the COVID-19 pandemic in Germany was influenced by two new major factors: detection of new virus Variants of Concern (VOC) and the start of the vaccination campaign. This study sought to evaluate the effect of these factors on in-hospital mortality in a multicenter study. Recently published results of the CORONA-Germany study showed temporal pattern of in-hospital mortality during the year 2020, influenced mostly by patients' age [1]. At this point in time, mutations of SARS-CoV-2 did not play a role as the first German patient with a VOC (202012/1; lineage B.1.1.7) was diagnosed on December 27th, 2020. However, from this time forward, frequency of B.1.1.7 increased and led to another rise of infection numbers in Germany from February to April 2021 with an increase of hospital admissions [2]. It was reported that the B.1.1.7 variant led to higher rates of severe and/or fatal courses of the disease [3,4], but influence on in-hospital mortality of COVID-19 patients is widely unknown. At the same time, the campaign started with vaccination of the elderly population ( $>80$ years), in particular with those living in nursing homes. From December 26th, 2020, until March 31st, 2021, five percent of the German population received full immunization [5].

\section{Methods}

We conducted a multicenter, observational, prospective, epidemiological cohort study at 45 hospitals in Germany, all from the same hospital network. Design and prior results of the CORONA-Germany-Study have been published previously [1]. In the current analysis, all hospitalized SARS CoV-2 positive patients were included with a complete hospital stay until April 19th, 2021. This study sought to evaluate the influence of new VOC as well as the vaccination campaign on in-hospital mortality and on the respiratory system evaluations by capturing mortality, the need for ventilation, respiratory failure, pneumonia, and mean age across time and presenting as moving averages. The estimated proportion of mutations (based on RKI data [2]) was adjusted by weekly age means and was related to mortality using a quasibinomial model. Odds ratios and $95 \%$ confidence intervals were shown. All p-values are two-sided. The significance level is $5 \%$. All analyses were performed with R (R Core Team 2021).

\section{Results}

A total of 10624 patients were included into the study (69+/18 yrs., 47\% female). Overall, 2021 (19\%) patients died during the hospital stay and 1558 (15\%) received mechanical ventilation for a median of 168 hours (IQR: 56, 371). Pneumonia was diagnosed in $58 \%(n=6198)$ and respiratory failure in 52\% $(n=5476)$. Further relevant diagnoses were atrial fibrillation, acute renal failure, and cardiac decompensation (Table 1). Figure 1A shows rates of SARS-
CoV-2 infections caused by B.1.1.7 and daily incidences (based on RKI data [2]) in relation to hospital admissions, showing an increase of hospital admissions correlating with increasing incidences and higher rates of B.1.1.7. In-hospital mortality showed temporal variations (Figure 1B). Age of hospitalized patients decreased until the end of the study from a mean age of approximately 73 years on December 31st, 2020, to about 63 years on April 19th, 2021 (Figure 1B). Age was related to mortality (Odds ratio \& 95\% confidence interval per year 1.086 [1.066, 1.107], p < 0.001), whereas the variant of concern SARS-CoV-2 B.1.1.7 had no association on inhospital mortality (10\% increase: 1.014 [0.990, 1.039], $\mathrm{p}=0.254$ ). Rates of pneumonia, respiratory failure and ventilation increased since October 2020, without a clear relationship to SARS-CoV-2 B.1.1.7.

Table 1: Patients characteristics of the CORONA-Germany study.

\begin{tabular}{|c|c|c|}
\hline Patients Characteristic & $\mathbf{N}$ & $(\mathrm{N}=10624)$ \\
\hline \multicolumn{3}{|c|}{ Baseline data } \\
\hline Age (yrs) & 10606 & $69( \pm 18)$ \\
\hline Sex (female) & 10624 & $5018 / 10624(47)$ \\
\hline \multicolumn{3}{|c|}{ Outcome data } \\
\hline Mortality & & $2021 / 10624$ (19) \\
\hline Ventilation & & $1558 / 10624(15)$ \\
\hline Hours of ventilation & 1558 & $168[56,371]$ \\
\hline Days of hospital stay & 10624 & $8[4,16]$ \\
\hline \multicolumn{3}{|c|}{ Diagnoses } \\
\hline ARDS & & $708 / 10624(6.7)$ \\
\hline Respiratory failure & & $5476 / 10624(52)$ \\
\hline Pneumonia & & $6198 / 10624(58)$ \\
\hline Cardiac decompensation & & $1142 / 10624(11)$ \\
\hline $\begin{array}{l}\text { Atrial fibrillation and atrial } \\
\text { flutter }\end{array}$ & & $2067 / 10624$ (19) \\
\hline Acute renal failure & & $1473 / 10624(14)$ \\
\hline Sepsis & & $917 / 10624(8.6)$ \\
\hline \multicolumn{3}{|c|}{ Therapies and procedures } \\
\hline Palliative care & & $1052 / 10624(9.9)$ \\
\hline ECMO & & $106 / 10624(1.0)$ \\
\hline Dialysis & & $399 / 10624(3.8)$ \\
\hline
\end{tabular}

Note: Continuous data are shown as means +- standard deviations or medians [ $25^{\text {th }}, 75^{\text {th }}$ percentiles]. Categorical data are shown as $\mathrm{N}$ occurrences / $\mathrm{N}$ totals (percentages). 


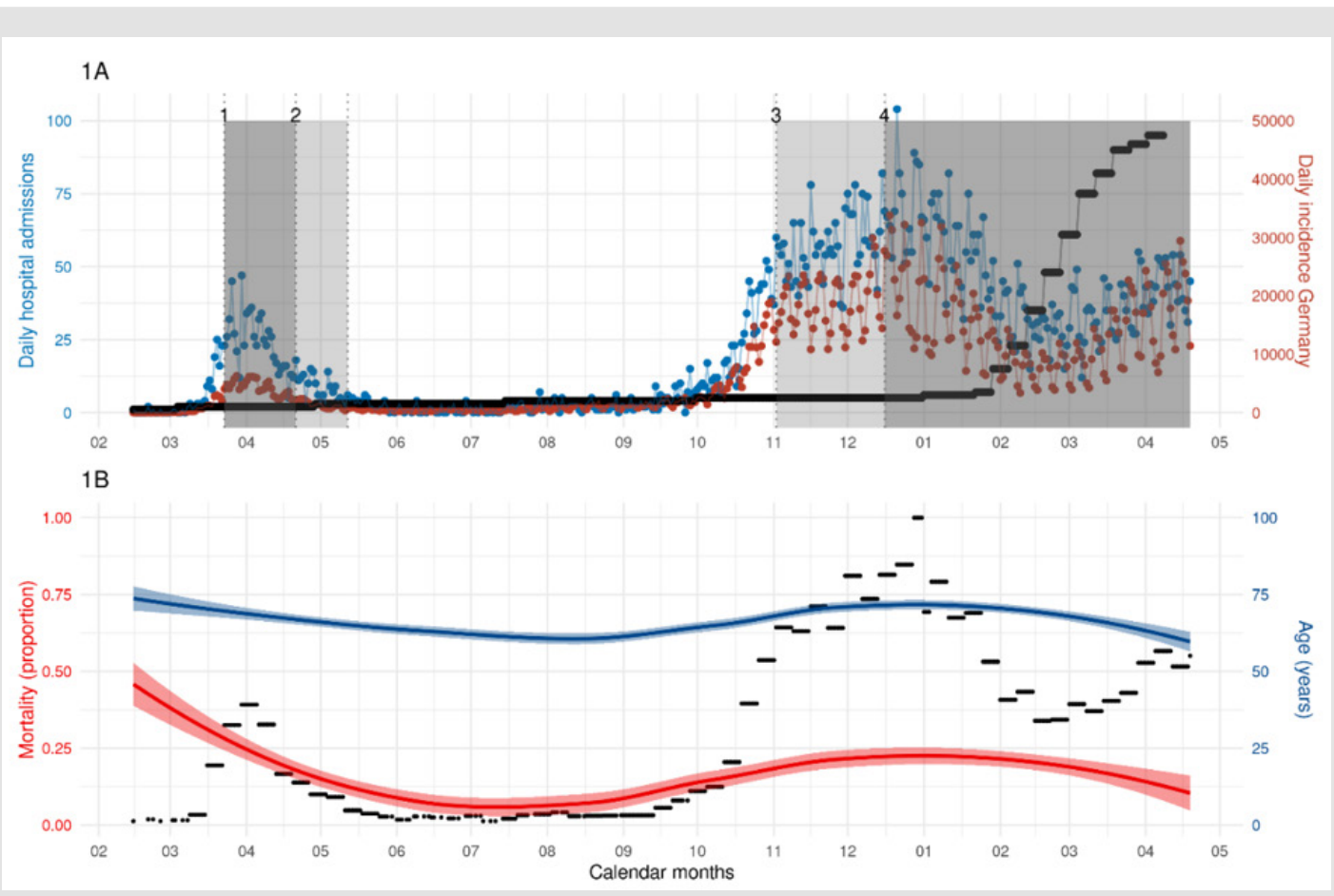

Figure 1:

a) Daily admissions of COVID-19 patients to the participating 45 centers in 2020/2021 (blue color), daily incidence of Germany in 2020/2021 (red color, data used with kind approval by RKI, [2]), and percentage of SARS-CoV-2 B.1.1.7 (black color, data used with kind approval by RKI, [2]) and is in accordance with the scale of the left (blue) vertical axis.

b) Temporal change of mortality (red color) and age (blue color) with 95\% confidence intervals. The black curve represents the proportion of weekly admissions of COVID cases with respect to the week with the most admissions (last week in 2020). As the values are scaled to $[0,1]$ the proportion of admissions is in accordance with the scale of the left (red) vertical axis.

\section{Discussion}

This new analysis of the CORONA-Germany cohort surprisingly shows that the occurrence of B.1.1.7 did not seem to influence in-hospital mortality. Despite increasing prevalence of the VOC B.1.1.7 in Germany, age is still the major risk factor on in-hospital mortality. Vaccination of the elderly most likely led to a shift in admission of a younger patient population to our hospitals, which may explain lower in-hospital mortality rate. Challen et al. reported in a British matched cohort study of 54,906 patients in each arm, that the risk of mortality was increased by infection with B.1.1.7 [3]. Other analyses of 2,245,263 positive SARS-CoV-2 community tests and 17,452 deaths associated with COVID-19 in England showed that B.1.1.7 is not only more transmissible than pre-existing SARS-CoV-2 variants but may also cause more severe illness and higher mortality [4]. Both study designs were different, including high proportions of outpatients with positive SARS-CoV-2 tests regardless of symptoms. This study focused on in-hospital patients only and had high proportions of moderate and severe ill COVID-19 patients with $58 \%$ of patients suffering from pneumonia and $52 \%$ from respiratory failure. Because of these selection differences, study results were not able to show the hazardous of B.1.1.7 in general, but they indicate that in patients who underwent hospital admission, existence of VOC B.1.1.7 did not affect in-hospital mortality. The major risk factor for in-hospital mortality was still patients' age.

\section{Limitation}

The proportion of SARS-CoV-2 B.1.1.7 infections on the study population was estimated by data available for the general population in Germany detected by the authorities (RKI). The occurrence of B.1.1.7 was adjusted by age, as it is the strongest risk factor for mortality. Other potential risk factors were not included in the analysis. The observational period of patients with a clinically relevant proportion of B.1.1.7. infections $>50 \%$ was only 6 weeks, which may have been too short to show an effect.

\section{Conclusion}

Despite a rise in new VOC (202012/1; lineage B.1.1.7) infections in Germany, in-hospital mortality did not increase. Age remained the major risk factor on mortality in hospitalized COVID-19 patients.

\section{Acknowledgment}

The authors thank the study teams for data entry, management and organization. Furthermore, we thank all the physicians and 
nurses from the COVID-19 wards of all participating centers for patient care during the ongoing pandemic.

\section{Conflict of Interest}

The authors have declared that no competing interests exist that could be perceived to bias this work. NG reports grants from Boston Scientific, grants from Medtronic and support from Bayer Vital outside the submitted work. JB reports personal fees from Actelion, AstraZeneca, Bayer, Biogen, BMS, Boehringer-Ingelheim, Galapagos, Novartis, Pliant, Sanofi-Genzyme, and Roche outside the submitted work. SW reports grants and personal fees from Abbott, Boston Scientific and Medtronic, and personal feels from Abbott, Boehringer Ingelheim, Bristol Myers Squibb, Bayer Vital, Acutus, and Daiichi Sankyo, outside the submitted work.

\section{ISSN: 2574-1241}

DOI: 10.26717/BJSTR.2021.38.006138

Nele Gessler. Biomed J Sci \& Tech Res

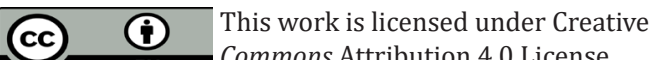

Submission Link: https://biomedres.us/submit-manuscript.php

\section{References}

1. Gessler N, Gunawardene MA, Wohlmuth P, Arnold D, Behr J, et al. (2021) Clinical outcome, risk assessment, and seasonal variation in hospitalized COVID-19 patients-Results from the CORONA Germany study. PLoS One 16(6): e0252867.

2. Robert Koch-Institut [Internet]: Accessed June $30^{\text {th }}, 2021$. Available from: https://www.rki.de/DE/Content/InfAZ/N/Neuartiges_Coronavirus/ Daten/Fallzahlen_Kum_Tab.html

3. Challen R, Brooks Pollock E, Read JM, Dyson L, Tsaneva Atanasova K, et al. (2021) Risk of mortality in patients infected with SARS-CoV-2 variant of concern 202012/1: matched cohort study. BMJ 372: n579.

4. Davies NG, Jarvis CI, Edmunds WJ, Jewell NP, Diaz Ordaz K, et al. (2021) Increased mortality in community-tested cases of SARS-CoV-2 lineage B.1.1.7. Nature 593(7858): 270-274.

5. Bundesministerium für Gesundheit [Internet]: Accessed at June $30^{\text {th }}$, 2021. Available from: https://impfdashboard.de

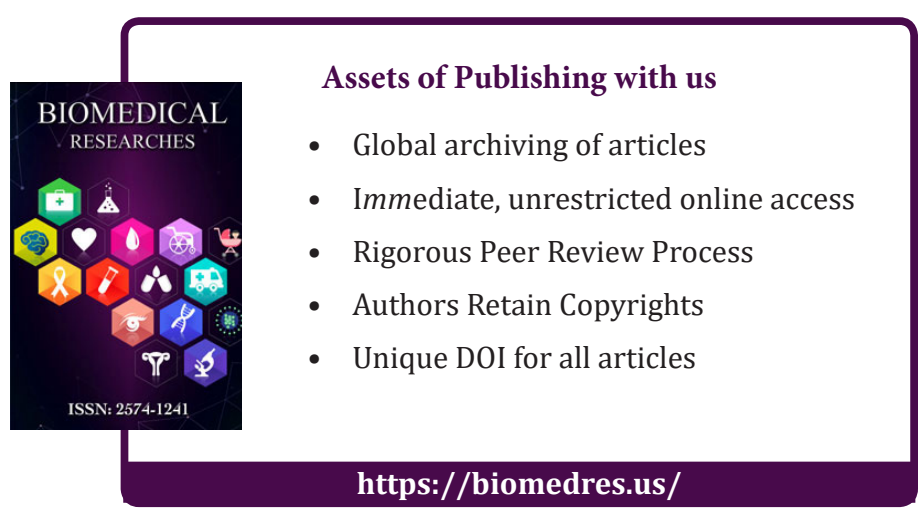

